\title{
THE UNDECIDABILITY OF THEORIES OF GROUPOIDS WITH AN EXTRA PREDICATE
}

\author{
SOLOMON GARFUNKEL AND JAMES H. SCHMERL
}

\begin{abstract}
Let $T$ be any theory in the language of groupoids, and let $T^{\prime}$ be the same theory considered now in the language with an extra unary predicate. If some model of $T$ has a substructure which is an infinite cancellative groupoid, then $T^{\prime}$ is hereditarily undecidable.
\end{abstract}

A groupoid is any structure $(B, \circ)$ in which $\circ$ is a binary operation defined on the set $B$. Suppose that $T$ is any first-order theory in the language of groupoids. Then let $T^{\prime}$ be the same theory as $T$ but now considered in the language with $\circ$ and the extra unary predicate $U$. Garfunkel [4] announced a proof of the fact that $T^{\prime}$ is hereditarily undecidable whenever $T$ is one of the following: the theory of finite elementary abelian groups, the theory of periodic abelian groups, or the theory of finite cyclic groups. His proof employed some involved codings. In this note we give a short proof of a very general theorem encompassing all of these results.

It should be remarked that all the results of [4] follow from a theorem announced by Isard [5] which states that if $T$ includes the theory of commutative semigroups and has a model with an element of infinite order, then $T^{\prime}$ is (apparently hereditrarily) undecidable. However, Garfunkel's proof did extend to some theories in which the orders of all the elements are uniformly bounded. (For instance, take $T$ to be the theory of abelian groups of exponent $p^{2}$ for some prime $p$.)

We call a groupoid $(B, \circ)$ a cancellative groupoid if it satisfies both the left and right cancellation laws: $\forall x y z(x \circ y=x \circ z \rightarrow y=z)$ and $\forall x y z(y \circ x=z \circ x \rightarrow y=z)$. A quasigroup is just a cancellative groupoid satisfying the additional axioms $\forall y z \exists x(x \circ y=z)$ and $\forall x z \exists y(x \circ y=z)$. By a theorem of Bates [1], cancellative groupoids are just those grou poids which can be embedded in quasigroups.

THEOREM. If some model of $T$ has a substructure which is an infinite cancellative groupoid, then $T^{\prime}$ is hereditarily undecidable.

Received by the editors January 27, 1973.

AMS (MOS) subject classifications (1970). Primary $02 \mathrm{G} 05$.

(c) American Mathematical Socie ty 1974 
Notice that the hypothesis of the theorem demands neither commutativity nor associativity. However, the hypothesis is obviously satisfied by any $T$ which has as a model an infinite group (or even quasigroup), or a semigroup with an element of infinite order, thereby implying Isard's theorem.

Proof. The proof relies on a theorem of [3] which is a modification of the Rabin-Scott technique [6] for proving undecidability. We restate the theorem here in a specialized form sufficient for our purpose:

Suppose that there exist formulas $\phi(x, v)$ and $\psi(x, y, v)$ in the language of $T^{\prime}$ with the property that for each countable, symmetric, irreflexive binary relational structure $(A, R)$, there is a model $(B, \circ)$ of $T$, a subset $U \subset B$ and an element $d \in B$ such that

where

$$
(A, R) \cong\left(A^{\prime}, R^{\prime}\right)
$$

and

$$
A^{\prime}=\{b \in B:(B, \circ, U) \vDash \phi(b, d)\}
$$

$$
R^{\prime}=\left\{\langle a, b\rangle \in B^{2}:(B, \circ, U) \vDash \psi(a, b, d)\right\} .
$$

Then $T^{\prime}$ is hereditrarily undecidable.

(Note. This combines the result of [3] with the fact that the theory of symmetric, irreflexive binary relational structures is hereditarily undecidable.)

The formulas $\phi(x, v)$ and $\psi(x, y, v)$, which we will show to satisfy the above hypothesis, are defined by

and

$$
\phi(x, v) \equiv U(x) \wedge \sim U(v \circ x)
$$

$$
\psi(x, y, v) \equiv \phi(x, v) \wedge \phi(y, v) \wedge U(x \circ y) \wedge x \neq y .
$$

We will now find a model $(B, \circ)$ of $T$ and an element $d \in B$ which will work for all choices of $(A, R)$. Let $(B, \circ)$ be any model of $T$ which has as a substructure an infinite cancellative groupoid, say $(C, \circ)$. Furthermore, by the familiar usage of Ramsey's Theorem and the Compactness Theorem, we can make the choice so that there is an indiscernible sequence $\left\langle b_{i}: i\langle\omega\rangle\right.$ of distinct elements of $C$. (Recall that the sequence $\left\langle b_{i}: i<\omega\right\rangle$ is indiscernible with respect to the formula $\theta\left(v_{0}, \cdots, v_{n-1}\right)$ iff whenever $i_{0}, \cdots, i_{n-1}, j_{0}, \cdots, j_{n-1}<\omega$ are such that $i_{r} \leqq i_{s}$ iff $j_{r} \leqq j_{s}$, then

$$
(B, \circ) \vDash \theta\left(b_{i_{0}}, \cdots, b_{i_{n-1}}\right) \leftrightarrow \theta\left(b_{j_{0}}, \cdots, b_{j_{n-1}}\right) .
$$

The sequence $\left\langle b_{i}: i\langle\omega\rangle\right.$ is indiscernible iff it is indiscernible with respect to all formulas.) We choose $d$ to be the element $b_{0}$. 
Now let $(A, R)$ be a countable, symmetric, irreflexive binary relational structure, where $A=\left\{a_{i}: 0<i<N\right\}$ for some $N \leqq \omega$. For any element $b \in B$ we inductively define $d^{m}(b)$ by setting $d^{0}(b)=b$ and $d^{m+1}(b)=d \circ d^{m}(b)$. Now let

$$
U=\left\{b_{i}: 0<i<N\right\} \cup\left\{d^{m}\left(b_{j} \circ b_{k}\right):\left\langle a_{j}, a_{k}\right\rangle \in R \text { and } m<\omega\right\} .
$$

Using the definitions of $A^{\prime}$ and $R^{\prime}$ as given before, we must now show that $(A, R) \cong\left(A^{\prime}, R^{\prime}\right)$. Indeed, what we will show is that

$$
A^{\prime}=\left\{b_{i}: 0<i<N\right\} \text { and } R^{\prime}=\left\{\left\langle b_{j}, b_{k}\right\rangle:\left\langle a_{j}, a_{k}\right\rangle \in R\right\} .
$$

We verify the first of the above equalities. Suppose $b \in A^{\prime}$, so that $b \in U$. Now if $b$ is not one of the $b_{i}$ for $0<i<N$, then $b=d^{m}\left(b_{j} \circ b_{k}\right)$ where $\left\langle a_{j}, a_{k}\right\rangle \in R$. But then $d \circ b=d^{m+1}\left(b_{j} \circ b_{k}\right) \in U$, contradicting the fact that $d \circ b \notin U$. Conversely, suppose that $b_{i} \notin A^{\prime}$ where $0<i<N$. Since $b_{i} \in U$ it must be that $d \circ b_{i} \in U$. This gives rise to two cases. In the first case $d \circ b_{i}=b_{j}$ where $0<j<N$. But, by indiscernibility with respect to the formula $v_{0}=v_{1} \circ v_{2}$, we get that $b_{j+1}=b_{0} \circ b_{i+1}=b_{1} \circ b_{i+1}$, and then by right cancellation $b_{1}=b_{0}$, which is a contradiction. In the second case $d \circ b_{i}=$ $d^{m}\left(b_{j} \circ b_{k}\right)$ where $m<\omega$ and $\left\langle a_{j}, a_{k}\right\rangle \in R$. Thus either $i \neq j$ or $i \neq k$. If $i \neq k$ then by indiscernibility with respect to the formula $v_{0} \circ v_{1}=v_{0}^{m}\left(v_{2} \circ v_{3}\right)$, we get that $d \circ b_{2 i}=d^{m}\left(b_{2 j} \circ b_{2 k}\right)=d^{m}\left(b_{2 j} \circ b_{2 k+1}\right)$, and then by left cancellation $b_{2 k}=b_{2 k+1}$, which is a contradiction. If $i \neq j$, then similarly $d^{m}\left(b_{2 j} \circ b_{2 k}\right)=d^{m}\left(b_{2 j+1} \circ b_{2 k}\right)$ leads to a contradiction by left and right cancellation.

Now to verify the second of the above equalities, suppose $\left\langle b, b^{\prime}\right\rangle \in R^{\prime}$. Then there are distinct $b_{r}, b_{s} \in A^{\prime}$ such that $b=b_{r}, b^{\prime}=b_{s}$ and $b_{r} \circ b_{s} \in U$. As before, it cannot be that $b_{r} \circ b_{s}=b_{i}$, so it must be that $b_{r} \circ b_{s}=$ $d^{m}\left(b_{j} \circ b_{k}\right)$, where $\left\langle a_{j}, a_{k}\right\rangle \in R$. If $\{r, s\}=\{j, k\}$, then $\left\langle a_{r}, a_{s}\right\rangle \in R$, so we can assume that either $k \neq r, s$ or $j \neq r, s$. If $k \neq r, s$, then, using indiscernibility again, we get that $b_{2 r} \circ b_{2 s}=d^{m}\left(b_{2 j} \circ b_{2 k}\right)=d^{m}\left(b_{2 j} \circ b_{2 k+1}\right)$, and then by left cancellation $b_{2 k}=b_{2 k+1}$, which is a contradiction. If $j \neq r, s$, then $d^{m}\left(b_{2 j+1}, b_{2 k}\right)=d^{m}\left(b_{2 j}, b_{2 k}\right)$ leads to a contradiction. Conversely, if $\left\langle b_{j}, b_{k}\right\rangle \notin R^{\prime}$, then it is clear that $\left\langle a_{j}, a_{k}\right\rangle \notin R$.

In [2] there is a list of 31 theories with an extra unary predicate which at that time were not known to be decidable (although most were known to be undecidable). The strength of our theorem is indicated by the fact that the undecidability of 28 of these can be obtained as immediate consequences of our theorem. Included among these are the theories of algebraically closed fields of a fixed characteristic, the theory of Boolean algebras (use symmetric difference) and theory of ordinal addition. The theory of infinite ordinal addition with an extra predicate can also be shown undecidable by considering the subgroupoid consisting of $\{\omega \cdot i: i<\omega\}$. 
We conclude with the following problem:

Problem. Characterize those $T$ for which $T^{\prime}$ is (hereditarily) undecidable. For example, is there some natural $T$ no model of which has an infinite cancellative subgroupoid and for which $T^{\prime}$ is undecidable?

ADDED IN PROOF. Only recently did it come to our attention that a result much stronger than Isard's has been proved by $\mathbf{R}$. McKenzie (Negative solution of the decision problem for sentences true in every subalgebra of $\langle N,+\rangle$, J. Symbolic Logic 36 (1971), 607-609). In that article, as the title indicates, it is proved that the theory of the class of subgroupoids of the semigroup of the natural numbers under addition is hereditarily undecidable. By using indiscernibles in a manner as we have done here, one can avoid the arithmetic computation at the end of McKenzie's proof.

\section{REFERENCES}

1. Grace E. Bates, Free loops and nets and their generalizations, Amer. J. Math. 69 (1947), 499-550. MR 9, 8.

2. Ju. L. Eršov, I. A. Lavrov, A. D. Taĭmanov and M. A. Taĭclin, Elementary theories, Uspehi Mat. Nauk 20 (1965), no. 4 (124), 37-108=Russian Math. Surveys 20 (1965), no. 4, 35-105. MR 32 \#4012.

3. S. Garfunkel, On the undecidability of certain finite theories, Trans. Amer. Math. Soc. (to appear).

4. - On the undecidability of certain classes of abelian groups with an extra unary predicate, Notices Amer. Math. Soc. 15 (1968), 632. Abstract \#68T-439.

5. S. Isard, Theories of algebraic structures with a distinguished subset, Notices Amer. Math. Soc. 14 (1967), 378. Abstract \#644-67.

6. M. Rabin, A simple method for undecidability proofs and some applications, Logic, Methodology and Philos. Sci. (Proc. 1964 Internat. Congr.), North-Holland, Amsterdam, 1965, pp. 58-68. MR 36 \#4976.

Department of Mathematics, University of Connecticut, Storrs, Connecticut 06268 